\title{
Comparison of pattern- onset, -reversal and -offset VEPs in treated amblyopia
}

FATIMA S. SHAWKAT, ANTHONY KRISS, CHRIS TIMMS, DAVID S.I. TAYLOR

\section{Abstract}

Purpose There are differences in the properties of visual evoked potentials (VEPs) to various forms of pattern stimulation and it is not clear how these differences reflect macular and parmacular function in amblyopic and normal eyes. We assessed pattern-onset, -reversal and -offset VEPs from amblyopic eyes and compared them with the responses from the fellow eyes, and from controls, to gauge the relative effectiveness of these stimulus modes.

Methods The three modes of pattern stimulation were presented sequentially in a single recording run to enable direct comparisons to be made for identical recording conditions. Half-field stimulation was used, as this elicits components over the ipsilateral and contralateral occipital scalp relative to the stimulated half-field, which reflect stimulation of macular and paramacular areas of the visual field. Eighteen amblyopes treated by occlusion and 20 control children were studied.

Results Multivariate analysis of variance showed significant differences between ambylopic and fellow eyes in amblyopes: pattern-onset components were significantly attenuated and ipsilateral reversal components were significantly prolonged in amblyopic eyes. When fellow eyes of amblyopes were compared with the eyes of controls, the reversal P100 and offset P110 and N165 components showed significant differences. Conclusions Ipsilateral reversal components and onset CII and contralateral P105 were the most affected in amblyopic eyes. The subnormal findings for the fellow eyes of amblyopes suggest that occlusion may have a long-standing physiological effect on the patched eye, not normally clinically apparent.

Key words Amblyopia, Macular, Paramacular, Pattern VEP

It is generally reported that amblyopic eyes tend to elicit reduced pattern visual evoked potential (VEP) amplitudes compared with fellow eyes.
Spekreijse et al. ${ }^{1}$ used pattern onset/offset stimulation with 20' checks and described that both onset and offset VEPs were much reduced in amplitude from the amblyopic eye when compared with the fellow eye. The onset/offset response of the amblyopic eye resembled that of the fellow eye elicited using an annulus (3-5 deg) stimulus field, and thus suggested that the VEP from the amblyopic eye had a predominant contribution from the macular region. The amblyopic eye also had a reduced VEP amplitude as compared with the fellow eye for a large range of checksizes (2' to $\left.100^{\prime}\right)$. The fellow eye showed tuning characteristics with the largest onset VEP amplitudes for 12' checks, while the amblyopic eye gave a maximal response for the larger 50' checks.

Sokol and Bloom ${ }^{2}$ compared monocular VEPs from non-amblyopic and amblyopic eyes in 15 subjects, using pattern reversal stimuli (15' checks, $18 \times 18$ deg display size, 12 reversals/s). All subjects had smaller VEPs from the amblyopic eye. Checksize effect was studied in one subject, who showed a maximum VEP amplitude for 15 ' checks from the nonamblyopic eye, whereas for the amblyopic eye 60 ' checks produced the largest responses. Arden and Barnard ${ }^{3}$ studied 28 normal and 71 amblyopic children, aged 4-11 years. Fifty-six of the amblyopes had undergone occlusion therapy. In the amblyopic eyes the latency of the VEP was increased and the amplitude was decreased, roughly in proportion to the loss of visual acuity; the VEP was normal in the nonamblyopic eyes that had never had occlusion therapy. Furthermore, occlusion affected the fellow eyes of amblyopes, and increased the VEP latency beyond that of the amblyopic eye latency. It was found that after the end of occlusion the VEP usually returned to normal; however, in 12 patients with prolonged occlusion, the change had not completely reversed to normal when tested 1 year later. Sokol ${ }^{4}$ measured the P100 latency of the pattern reversal VEP for small checks (15') in 68 normal and 32 amblyopic children. In normal children there were no significant interocular latency
F.S. Shawkat

A. Kriss

C. Timms

D.S.I. Taylor

Department of

Ophthalmology

Great Ormond Street

Hospital for Children

London, UK

F.S. Shawkat

Department of

Ophthalmology

Great Ormond Street

Hospital for Children NHS

Trust

Great Ormond Street

London WC1 3JH, UK

Tel: +44 (0)1714059200,

ext $0251 / 8651$

Fax: +44 (0)171 8298647 
differences, but the amblyopic children showed longer P100 latencies in their amblyopic eye compared with their fellow eye.

VEP studies of amblyopia have demonstrated attenuation of VEPs, mainly to macular-derived, small check (high spatial frequency) stimulation. A comparison of pattern-onset, -reversal and -offset modes of stimulation, using half-field stimulation to elicit ipsilateral and contralateral half-field components, appears not to have been previously investigated in amblyopes. Half-field stimulation was adopted to separate contributions from macular and paramacular areas of the visual field. It is known that when the whole full-field is used, components of opposite polarity algebraically summate and are either not detectable or very difficult to discern. ${ }^{5}$ In particular, those components recorded from the contralateral side of the scalp to the stimulated half-field, namely reversal N105, offset N115 and onset P105, are not well seen for full-field stimulation. We have investigated previously the effects of checksize ${ }^{6}$ and experimental scotomata ${ }^{7}$ on patternonset, -reversal and -offset components in healthy controls with normal binocular vision. In this study we assess the relative clinical efficacy of different modes of pattern stimulation in amblyopic patients. Results concerning binocular interaction of pattern VEPs in this group of amblyopic patients have been reported fully elsewhere. ${ }^{8}$

\section{Subjects and methods \\ Subjects}

The amblyopic subject group consisted of 18 treated amblyopes, aged 7-16 years (mean 11 years; 7 males, 11 females) who were compared with 20 healthy control children with normal binocular vision, aged 6-16 years (mean 11 years; 12 males, 8 females).

Control subjects had corrected Snellen acuities of $6 / 5$ or better with no history of visual problems. Amblyopic children had a full orthoptic examination prior to electrophysiological testing. Fourteen were strabismic amblyopes (esotropes) and 4 were anisometropic amblyopes. All strabismic amblyopes had convergent squints at the time of VEP recording. Corrected acuities of the non-amblyopic eyes at the time of recording were $6 / 5$ or $6 / 6$, with the exception of 2 strabismic patients, whose acuities were $6 / 9$. Corrected acuities of the amblyopic eyes ranged from $6 / 36$ to $6 / 6$ (mean acuity $6 / 12$ ). Binocular single vision (assessed using the Randot stereotest) was absent in 13 patients (72.2\%) and only grossly present in the remaining 5 cases (Randot to 200 seconds of arc). None had latent or manifest latent nystagmus.

All the amblyopic children had undergone occlusion therapy with good levels of compliance. Age at which occlusion was started ranged from 2 to 6 years (mean 4.2 years), and the mean number of years of patching was 2.5 years. The extent of occlusion was somewhat variable but tended to be for longer periods in the first few months of therapy (e.g. 6 hours per day) compared with later (e.g.
$1 / 2$ hour per day). Only one patient was still having patching therapy of 1 hour per day at the time of VEP recording, whereas the remaining amblyopes had ended their occlusion therapy at least 2 years prior to testing. The variability in occlusion history of the amblyopes did not lend itself to statistical analysis with respect to VEP measures.

\section{Technique}

Occipital VEPs were recorded using EEG silver/silver chloride electrodes, attached to the scalp with collodion. Impedance was reduced by gentle skin scarification to $<10 \mathrm{k} \Omega$. A seven-channel montage was used; this consisted of a tranverse row of five electrodes placed $5 \mathrm{~cm}$ above the inion and $5 \mathrm{~cm}$ apart, so that two electrodes were spaced at 5 and $10 \mathrm{~cm}$ to either side of the midline electrode. Electrodes were also placed at the inion, and $2.5 \mathrm{~cm}$ above the inion. All occipital electrodes were referred to a common mid-frontal reference $\left(\mathrm{F}_{\mathrm{z}}\right)$ and the ground electrode was placed at the vertex.

Two oscilloscopes (Hewlett Packard, 1321A, X-Y Display, $\mathrm{P} 4$ phosphor), each subtending 24 deg by $18.5 \mathrm{deg}$, were clamped together, at a distance of $1 \mathrm{~m}$ from the subject. These were viewed through a mirror stereoscope so that the subject's left eye viewed one oscilloscope and the right eye viewed the other oscilloscope. Black and white checkerboard patterns were presented in the left half-field (0-12 deg) of each oscilloscope screen; the right half consisted of a uniform grey field of the same average luminance as the checkerboard. A small ring at the centre of the vertical border of the pattern/blank interface provided a fixation spot. The luminance levels were $11.5 \mathrm{~cd} / \mathrm{m}^{2}$ for white squares and $0.004 \mathrm{~cd} / \mathrm{m}^{2}$ for black squares, and were constant across checksizes. Four checksizes were used subtending 12, 20, 50 and 80 minutes of arc at the subject's eye. Binocular, left and right eye responses for each of the four checksizes were recorded; the test order was randomly varied from subject to subject. All control and amblyopic children were recorded wearing correct spectacle correction when necessary.

VEPs to pattern-onset, -reversal and -offset were acquired sequentially in a single epoch. Details regarding this mode of stimulation have been described in previous studies. ${ }^{6-8}$ A 20 ms pre-stimulus interval preceded the stimulus sequence, after which the checkerboard appeared for $300 \mathrm{~ms}$, was reversed for the subsequent $300 \mathrm{~ms}$, and then disappeared for $300 \mathrm{~ms}$ (pattern-offset). Previous studies using a similar stimulus paradigm have shown that $300 \mathrm{~ms}$ is a sufficient time interval between successive contrast changes to obtain reliable responses to the three stimulus modes. ${ }^{9}$ An average of 100 such sequences was recorded for each checksize and viewing condition. When necessary averaging was repeated, for example, if the subject attended poorly, or the responses were corrupted by myogenic or electrode movement artefacts. 
IPSILATERAL

CHANNEL 2

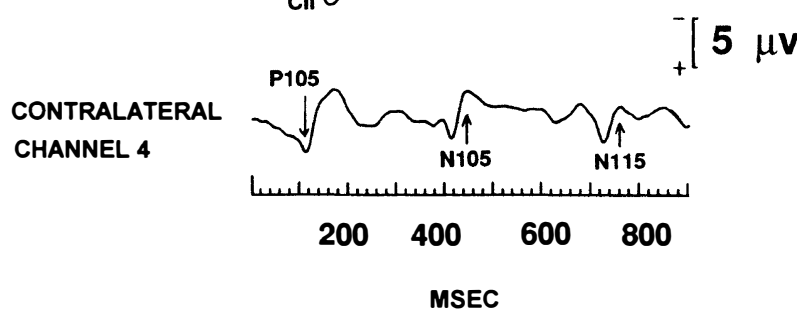

Fig. 1. Pattern VEPs to 50' checks of a control subject. Left half-field pattern-onset, -reversal and-offset components were recorded from the ipsilateral ( $5 \mathrm{~cm}$ to the left of the midline electrode) and contralateral channels ( $5 \mathrm{~cm}$ to the right of the midline electrode).

A PDP11 computer was used for averaging responses. Each input channel had a setting of $1 \mathrm{~s}$ time constant and a high-frequency cut-off of less than $3 \mathrm{~dB}$ at $80 \mathrm{~Hz}$. The input signal was digitised with a sampling rate of 0.5 points/ms.

Ipsilateral and contralateral half-field occipital VEPs were best seen in the $5 \mathrm{~cm}$ lateral channels (in agreement with other studies ${ }^{10}$ ). Therefore, components were measured from the electrodes $5 \mathrm{~cm}$ to the left and to the right of the midline. Component recognition was based on their polarity and latency range (see Jeffreys, ${ }^{11}$ Halliday et al., ${ }^{12}$ and Kriss and Halliday ${ }^{13}$ for details regarding component identification). The following VEP components were measured: for pattern-onset, the first three ipsilateral components CI, CII, CIII and contralateral P105; for reversal, the ipsilateral N80, P100, N145 and contralateral N105; and for offset, the ipsilateral N85, P110, N165 and contralateral N115 (Fig. 1). Peak latency and peak-to-peak amplitude measurements were made for each of these components.

\section{Results}

Comparison of amblyopic with fellow eyes in amblyopic children

For the majority of VEP components, amplitudes were smaller and latencies more prolonged from the amblyopic eye when compared with the fellow eye. Multivariate analysis of variance (MANOVA) of the onset, reversal and offset VEPs with respect to checksize and viewing eye showed significant differences related to amblyopia (Pillais test, $F=2.159, p<0.012$ ) and checksize $(F=2.88, p<0.0001)$, although checksize by viewing eye was not significant $(F=0.7, p=0.88)$. Fig. 2 shows the binocular, amblyopic and fellow eye ipsilateral and contralateral responses from an amblyopic child.

Univariate analysis showed that only some VEP components contributed to the effect of viewing eye: namely pattern-onset CII and contralateral P105 amplitudes and all ipsilateral pattern-reversal components (Table 1). Amblyopic eyes were more prolonged than fellow eyes in the range of 5-12 ms for P100 (mean $=9 \mathrm{~ms}), 4-12 \mathrm{~ms}$ (mean $=8 \mathrm{~ms})$ for N80 and 7-13 ms (mean = $11 \mathrm{~ms})$ for N145. The P100 amplitude of

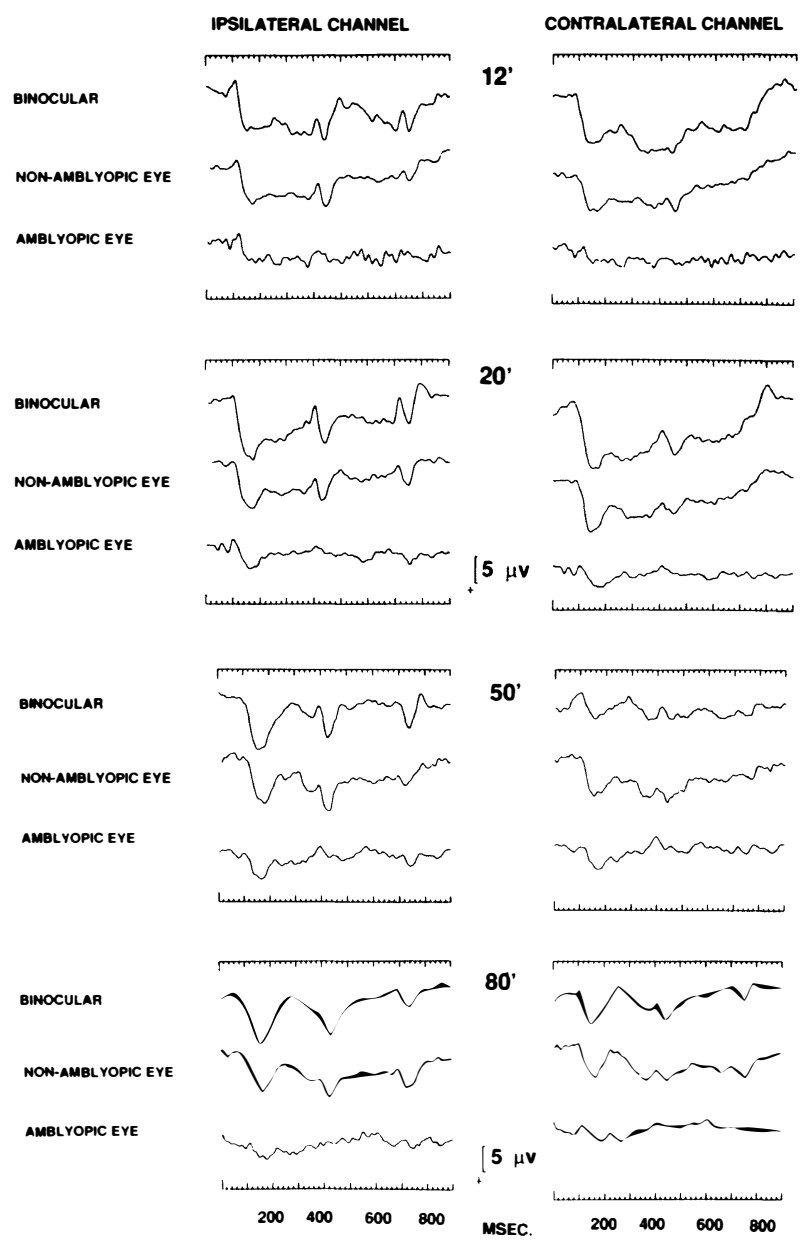

Fig. 2. Binocular and monocular ipsilateral and contralateral responses to different checksizes from an 11-year-old amblyopic subject.

fellow eyes showed a tuning effect with the largest responses occurring for 50 ' checks $(5.6 \mu \mathrm{V} \pm 1.8)$; however, amblyopic eye amplitudes were largest for the largest 80' checks, and at this checksize the mean amblyopic eye amplitude was greater than that of the fellow eye (amblyopic eye: $5.3 \mu \mathrm{V} \pm 3.3$; fellow eye: $3.6 \mu \mathrm{V} \pm 2.6)$.

Binocular results relating to interocular interaction have been reported elsewhere. ${ }^{8}$ Essentially, interocular interaction in normals was best seen for potentials that predominantly reflect macular pathway activity, and were most conspicuous for reversal N80 and P100 components. Binocular:monocular amplitude ratios for normals were compared with 'binocular:good eye' amplitude ratios for amblyopes. The reversal P100 ratio

Table 1. Comparison of amblyopic with fellow eyes: univariate analysis of VEP components that showed a significant effect

\begin{tabular}{lcc}
\hline Component & F value & $p$ value \\
\hline Pattern-onset & & \\
Ipsilateral CII amplitude & 6.87 & $<0.02$ \\
Contralateral P105 amplitude & 5.46 & $<0.03$ \\
Pattern-reversal & & \\
Ipsilateral N80 latency & 9.83 & $<0.001$ \\
Ipsilateral P100 latency & 14.64 & $<0.001$ \\
Ipsilateral N145 latency & 9.04 & $<0.005$ \\
\hline
\end{tabular}


Table 2. Comparison of amblyopic with control eyes: univariate analysis of VEP components that showed a significant effect

\begin{tabular}{lcl}
\hline Component & F value & $p$ value \\
\hline Pattern-onset & & \\
Ipsilateral CII amplitude & 4.9 & $<0.01$ \\
Ipsilateral CIII amplitude & 7.1 & $<0.001$ \\
Contralateral P105 amplitude & 6.3 & $<0.01$ \\
Contralateral P105 latency & 8.3 & $<0.001$ \\
Pattern-reversal & & \\
Ipsilateral N145 amplitude & 4.3 & $<0.05$ \\
Ipsilateral N80 latency & 19.3 & $<0.001$ \\
Ipsilateral P100 latency & 30.4 & $<0.001$ \\
Ipsilateral N145 latency & 13.3 & $<0.001$ \\
Pattern-offset & & \\
Ipsilateral N165 latency & 6.1 & $<0.02$ \\
\hline
\end{tabular}

was found to differ significantly between normals and amblyopes for 12', 20' and 50' checks. Ipsilateral (CII) and contralateral (P105) onset components also differed significantly, but for the smallest 12 ' checks only. In controls, onset components (P105 and CIII) and reversal components (N80 and P100) showed significantly shorter binocular as compared with monocular latencies. These latency differences were not found in amblyopes.

\section{Comparison of amblyopes and normally sighted subjects}

The VEP measurements from the amblyopic and fellow eyes of ambylopic children were compared with the eyes of control, normally sighted children of the same age range. MANOVA showed significant differences $(F=3.5$, $p<0.001)$ between the amplitudes of the amblyopic eye and control eye and this was attributable to the majority of components (Table 2). At least for the reversal P100 amplitude using 50' checks, $89 \%$ of amblyopic eye responses were smaller than the mean amplitude of control eyes $(7.2 \mu \mathrm{V} \pm 2.9)$. Fig. 3 is an example of binocular and monocular ipsilateral and contralateral responses from a control child.

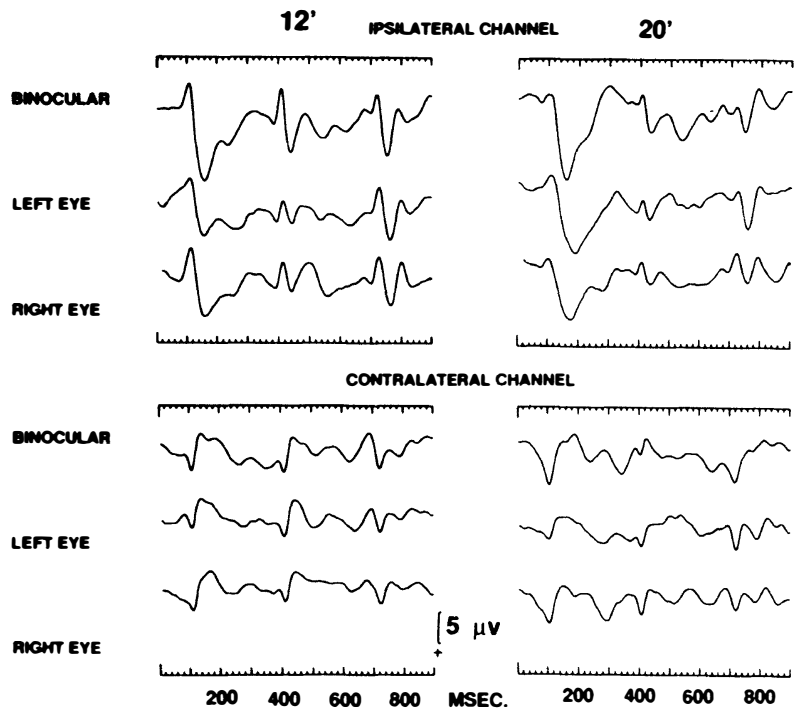

Interestingly, there were also significant differences when the fellow eyes of the amblyopic children were compared with control normal eyes (MANOVA: $F=3.0$; $p<0.001)$, although univariate analysis showed that these were only significant for reversal P100 amplitude $(p<0.05)$, offset P110 amplitude $(p<0.03)$ and offset N165 latency $(p<0.01)$. Fig. 4 shows that the P100 amplitude graph for the fellow eye is essentially of similar shape to that of the control eyes but there is a consistent difference in amplitude between the fellow eyes of amblyopes and controls. Indeed $83 \%$ of fellow eye P100 amplitudes for 50' checks were smaller than the mean for control eyes $(7.2 \mu \mathrm{V} \pm 2.9)$, which is comparable to our laboratory lower limits of normal used for routine clinical testing. A tuning effect for the reversal P100 amplitude occurs, so that maximum amplitudes are found for 50' checks; however, values for fellow eyes of amblyopes are consistently of smaller amplitude (Fig. 4). The offset P110 amplitude behaves in a similar manner to the reversal P100 and the latencies for offset N165 for the fellow eyes were consistently more prolonged than those of control eyes across all checksizes.

\section{Discussion}

There were more VEP components showing significant differences in amplitude and latency when amblyopic eyes were compared with the corresponding eyes of control subjects, as opposed to a within-subject comparison of amblyopic eyes versus fellow eyes. Only onset ipsilateral CII and contralateral P105 were significantly attenuated in the amblyopic eyes when compared with fellow eyes in the amblyopic subjects. On the other hand, reversal ipsilateral N80, P100 and N145 were significantly prolonged from the amblyopic eyes when compared with their fellow eyes. If VEPs from amblyopic eyes are compared with VEPs from control subjects, then as well as the above-mentioned
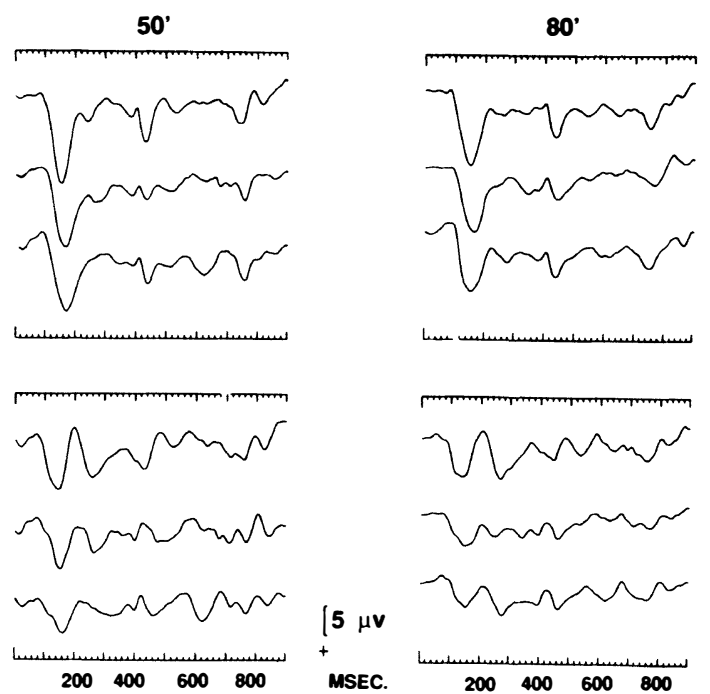

Fig. 3. Binocular and monocular ipsilateral and contralateral VEP responses of a normal 11-year-old. 


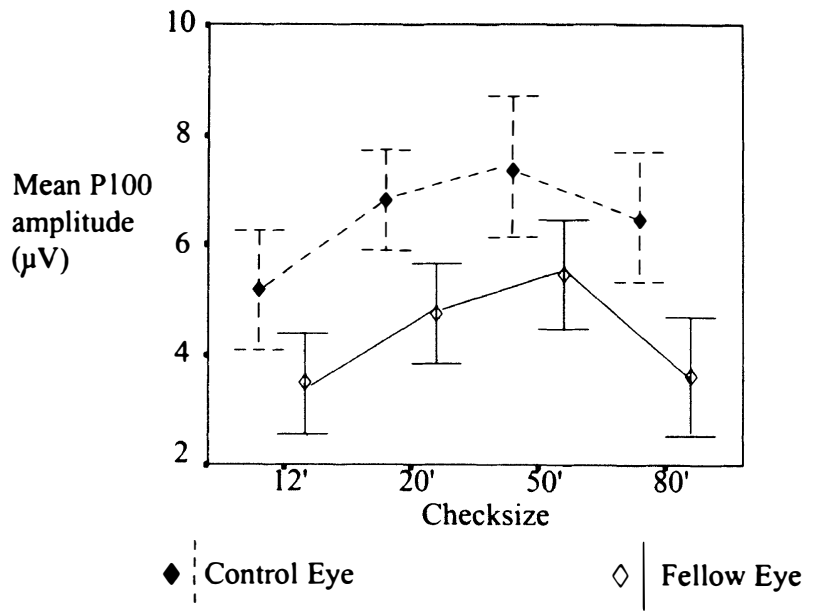

Fig. 4. Mean P100 amplitude (mean \pm SE) for fellow eyes of amblyopes (dashed lines) and normal control eyes (continuous lines) for the different checksizes.

components, onset CIII and reversal N145 amplitudes are also significantly attenuated, and onset (CI, CIII, P105) and offset N165 components are prolonged.

These differences, and the fact that significant differences were also found between the fellow eyes of amblyopic subjects and corresponding eyes of controls, indicate that the fellow eye of amblyopes also shows mildly 'abnormal' features. Although it is possible that these abnormal features were present prior to any treatment, ${ }^{14-16}$ we have evidence that this may not always be the case, as we have other data from patched and unpatched unilateral cataract cases which indicate that, in deprivational amblyopia at least, patching may have deleterious effects on the pattern VEP of fellow eyes. ${ }^{17,18}$ Arden and Barnard ${ }^{3}$ found that VEPs were larger and of shorter latency in the fellow eyes of amblyopes who have never had occlusion therapy, compared with those who had been patched. They found that occlusion amblyopia appeared to be reversible to a certain extent: amblyopic fellow eyes tested more than 3 months after the end of their occlusion therapy had similar VEPs to normals when using larger 46' and 23' checks; however, if small 11.5' checks were used then the fellow eye VEPs were significantly prolonged when compared with normals. All but one of our amblyopes had completed their occlusion therapy 2 or more years prior to VEP testing, yet we still found that reversal P100 and offset P110 and N165 were affected in the fellow eyes. Furthermore, unlike Arden and Barnard, ${ }^{3}$ who found that the long-term VEP deficits were confined to smaller checks, our results show that the VEPs were affected across all the checksizes, suggesting that occlusion amblyopia may have a longer-lasting, irreversible physiological effect. It is interesting to note that whereas amblyopic eyes lose their tuning effect for the reversal P100 amplitude (amplitude increases with increasing checksize), the fellow eye maintains a tuning effect similar to that in control eyes (Fig. 4: graphs are similar with amplitude maxima at 50' checks) but shifted downwards in amplitude.
The subnormal amplitude and latency findings for the fellow patched eyes of amblyopes are interesting and worthy of more detailed investigations. Our findings suggest that amblyopic patients undergoing occlusion therapy should have not only the amblyopic eye but also the patched fellow eye carefully assessed in order to minimise possible amblyopic effects associated with occlusion therapy. This effect may not be readily apparent on clinical testing, as all except 2 amblyopes had normal fellow eye Snellen acuities (yet $83 \%$ of these fellow eyes had VEP amplitudes smaller than average). It is important not only to compare amblyopic with fellow eyes, but also to compare both eyes of amblyopic patients with those of age-matched laboratory controls with normal acuity and binocular function. We are at present investigating visual function of the patched fellow eye in congenital unilateral cataract and using contrast sensitivity, transient and sweep VEP measures.

The reversal P100 component is thought to reflect predominantly macular pathway activity. ${ }^{19-21}$ Using a similar experimental paradigm, ipsilateral reversal components have been reported to be sensitive to checksize and were larger in amplitude and more prolonged in latency when elicited by small checksizes. ${ }^{6}$ Furthermore, the reversal P100 component was particularly sensitive to a small (0-1.5 deg) central scotoma. $^{7}$

Our results agree with previous reports of attenuated pattern-reversal and -onset VEPs from amblyopic eyes ${ }^{1,2,22,23}$ and suggest that the predominant contribution to the amblyopic eye VEP is from the paramacular areas. This is most clearly seen when comparing amblyopic and fellow eye changes of P100 VEP amplitude with checksize: the fellow eye shows a tuning effect with largest amplitudes at 50' check stimulation, whereas amblyopic eyes show a steady increase in amplitude with increasing checksize, so that the largest responses are found for the largest $80^{\prime}$ checks. In fact, at this checksize, mean amblyopic eye amplitudes are greater than that for the fellow eye. A similar finding was reported by Sokol and Bloom ${ }^{2}$ in one subject, where VEPs to 60 ' checks were greater in the amblyopic eye than in the fellow eye.

Sokol $^{24}$ used experimental scotomata and different field sizes to test amblyopic and normal eyes. He concluded that the VEP from an amblyopic eye arises from outside the central $3 \mathrm{deg}$ of the visual field. It has been shown that the central 1 deg field contributes to onset CII, and that P105 is derived from the central 2-6 deg; also CII and CIII have been found to depend less on contrast and more on pattern detail. ${ }^{25-27}$ Furthermore, results from experimental scotoma studies ${ }^{7}$ show that a central 0-1.5 deg scotoma significantly attenuated reversal P100 and N145 as well as onset contralateral P105. On the basis of the above results we expected onset CII, CIII, contralateral P105, reversal P100 and N145 to be affected in amblyopic eyes. On the other hand, onset $\mathrm{CI}$ is predominantly a luminance-dependent component, ${ }^{25-27}$ and it is maximal with the largest checksizes. ${ }^{6}$ The contralateral reversal N105 and offset N115 were not 
significantly affected by central scotoma ${ }^{7}$ and were maximal to the largest checksize, and were thus primarily of paramacular origin. ${ }^{6}$ It was postulated that onset CI and the contralateral components N105 and N115 primarily reflect paramacular activity, and thus we would not expect them to show any difference between normal and amblyopic eyes.

On the whole, findings from this study support these expectations. The amplitudes of onset CII, CIII and P105, reversal N145 and offset P110 and N165 were significantly attenuated in amblyopic eyes. Offset P110 and N165 have been postulated to be of similar origin to reversal P100 and N145, ${ }^{13,28}$ and thus would be expected to be affected in a similar manner in amblyopia.

Significantly prolonged VEP latencies in amblyopic eyes occurred for all ipsilateral reversal components, and for N85 and N165 offset components.

Prolonged and broadened onset contralateral P105 was found in amblyopic eyes. This possibly represents the paramacular 'subcomponent' of P105 that has been previously described. ${ }^{6,7}$ The contralateral P105 component has been shown to be sharply defined for smaller checksize $\left(\geqslant 35^{\prime}\right)$, but becomes broadened and bifid for larger checksizes $\left(50^{\prime}-110^{\prime}\right){ }^{6}$ It was postulated that the first limb of this bifid waveform corresponds to the well-defined positivity obtained with small checks, whereas the second positive limb of the waveform (occurring some $20 \mathrm{~ms}$ later) is a component specific for large checks and thus presumably of paramacular origin. Furthermore, introduction of an experimental central scotoma attenuated the sharply defined P105 to small checks but did not significantly influence the broad and bifid P105 when large checks were used. ${ }^{7}$ In our amblyopic subjects, the sharply defined early macular P105 seen with small check stimulation in normal eyes would not be produced by amblyopic eyes where macular vision is compromised.

In conclusion, the pattern VEP changes found in amblyopic eyes indicate deficits primarily affecting the central field of vision resulting in both the attenuation and prolongation of certain macular-derived VEP components. Ipsilateral pattern-reversal and -offset components were compromised in amblyopic eyes. Onset components were also affected, and this was particularly conspicuous for the macular-derived CII and contralateral P105 components. If amblyopic eyes are compared with fellow eyes, then pattern-reversal stimulation is the best mode for accentuating the differences between the affected and unaffected eyes.

However, when comparing an amblyopic eye with the normal eye of a control subject, then all ipsilateral and contralateral onset, and all ipsilateral reversal and offset components are affected. The significant difference between the fellow eyes of amblyopes and controls suggests occlusion therapy may be having a protracted effect on the patched fellow eye in amblyopes.

We would like to thank The Iris Fund for their financial support and Mr Jack Pitman for technical help.

\section{References}

1. Spekreijse H, Khoe LH, van der Tweel LH. A case of amblyopia: electrophysiology and psychophysics of luminance and contrast. In: Arden GB, editor. The visual system. New York: Plenum Press, 1972:141-56.

2. Sokol S, Bloom B. Visually evoked cortical responses of amblyopes to a spatially alternating stimulus. Invest Ophthalmol Vis Sci 1973;12:936-9.

3. Arden GB, Barnard WM. Effect of occlusion on the visual evoked response in amblyopia. Trans Ophthalmol Soc UK 1979;99:419-26.

4. Sokol S. Abnormal evoked potential latencies in amblyopia. Br J Ophthalmol 1983;67:310-4.

5. Blumhardt LD, Halliday AM. Hemisphere contributions to the composition of the pattern-evoked waveform. Exp Brain Res 1979;36:53-69.

6. Shawkat FS, Kriss A. Sequential pattern-onset, -reversal and -offset VEPs: comparison of effects of checksize. Ophthalmic Physiol Opt 1998;18:495-503.

7. Shawkat FS, Kriss A. Effects of experimental scotomata on sequential pattern-onset, -reversal, and -offset VEPs. Doc Ophthalmol 1998;94 (in press).

8. Shawkat FS, Kriss A. Interocular interaction assessed by VEPs to pattern-onset, -reversal and -offset in normally sighted and amblyopic subjects. Electroencephalogr Clin Neurophysiol 1997;104:74-81.

9. Kriss A, Spekreijse H, Verduyn Lunel HFE, Braamhaar I, de Waal BJ, Barrett G. A comparison of pattern onset, offset and reversal responses: effects of age, gender and check size. In: Nodar R, Barber C, editors. Evoked potentials II. New York: Butterworth, 1984: 553-61.

10. Barrett G, Blumhardt LD, Halliday AM, Halliday E, Kriss A. A paradox in the lateralization of the visual evoked response. Nature 1976;261:253-5.

11. Jeffreys DA. Cortical source locations of pattern-related VEPs (visual evoked potentials) recorded from the human scalp. Nature 1971;229:502-4.

12. Halliday AM, Barrett G, Blumhardt LD, Kriss A. The macular and paramacular subcomponents of the pattern evoked response. In: Lehmann D, Callaway E, editors. Human visual evoked potentials: applications and problems. New York: Plenum Press, 1979:135-51.

13. Kriss A, Halliday AM. A comparison of occipital potentials evoked by pattern onset, offset and reversal by movement. In: Barber C, editor. Evoked potentials. Lancaster: MTP Press, 1980:205-12.

14. Leguire LE, Rogers GL, Bremer DL. Amblyopia: the normal eye is not normal. J Pediatr Ophthalmol Strabismus 1990;27:32-8.

15. Zhao KX. An investigation of the multi-channel VEP topography by full field stimulation in functional amblyopia of children. Chung Hua Yen Ko Tsa Chih 1990;26:68-72.

16. Fil'chikova LI, Kriukovskikh ON, Dubovskaia LA, Matveev SG. The effect of occlusion of the better seeing eye on the function of the visual system in children with unilateral amblyopia. Vestn Oftalmol 1993;109:8-11.

17. Kriss A, Thompson DA, Lloyd IC, Jeffrey B, Russell-Eggitt I, Taylor D. Pattern VEP findings in young children treated for unilateral congenital cataract. In: Cottlier E, editor. Congenital cataract. Austin: RJ Landes, 1994:79-88.

18. Thompson DA, Møller H, Russell-Eggitt I, Kriss A. Visual acuity in unilateral cataract. Br J Ophthalmol 1996;80:794-8.

19. Blumhardt LD, Barrett G, Halliday AM, Kriss A. The effect of experimental 'scotomata' on the ipsilateral and contralateral responses to pattern reversal in one half-field. Electroencephalogr Clin Neurophysiol 1978;45:376-92.

20. Haimovic IC, Pedley TA. Hemi-field pattern reversal visual evoked potentials. I. Normal subjects. Electroencephalogr Clin Neurophysiol 1982;54:111-20. 
21. Yiannikas C, Walsh JC. The variation of the pattern shift visual evoked response with size of the stimulus field. Electroencephalogr Clin Neurophysiol 1983;55:427-35.

22. Arden GB, Barnard WM, Mushin AS. Visually evoked responses in amblyopia. Br J Ophthalmol 1974;58:183-92.

23. Regan D. Speedy assessment of visual acuity in amblyopia by the evoked potential method. Ophthalmologica 1977;175:159-64.

24. Sokol S. Visual evoked potentials to checkerboard pattern stimuli in strabismic amblyopia. In: Desmedt JE, editor. Visual evoked potentials in man: new developments. Oxford: Clarendon Press, 1977:410-7.
25. Jeffreys DA. Cortical source locations of pattern-related VEPs (visual evoked potentials) recorded from the human scalp. Nature 1971;229:502-4.

26. Jeffreys DA, Axford JG. Source locations of pattern-specific components of human visual evoked potentials. I. Component of striate cortical origin. Exp Brain Res 1972;6:1-21.

27. Jeffreys DA, Axford JG. Source locations of pattern-specific components of human visual evoked potentials. II.

Component of extrastriate cortical origin. Exp Brain Res 1972;6:22-40.

28. Estéves $\mathrm{O}$, Spekreijse H. Relationship between pattern appearance-disappearance and pattern reversal responses. Exp Brain Res 1974;19:233-8. 